\title{
aNAC inhibition of the FADD-JNK axis plays anti-apoptotic role in multiple cancer cells
}

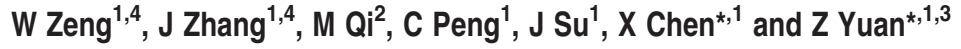

Nascent polypeptide-associated complex $\alpha$ ( $\alpha \mathrm{NAC})$ is reportedly overexpressed in several types of cancers and regulates cell apoptosis under hypoxic conditions in HeLa cells. The aim of our study was to investigate the apoptotic function of $\alpha$ NAC in cancer progression. First, we observed the cellular effects of $\alpha$ NAC depletion. Mouse $\alpha$ NAC was used to restore the protein level and verify the effect. An Annexin V assay, a caspase activity reporter assay, an apoptotic molecular marker, and a colony formation assay were used as markers to investigate the mechanisms of cell death caused by aNAC depletion. The Cancer 10-pathway reporter assay was used to screen downstream pathways. PCR site-directed deletion based on the functional domains of $\alpha$ NAC was used to construct deletion mutants. Those functional domain deletion mutants were used to recover the apoptotic phenotype caused by $\alpha$ NAC depletion. Finally, the role of $\alpha$ NAC in TNF-related apoptosis-inducing ligand (TRAIL) treatment was investigated in vitro. We found that depletion of $\alpha$ NAC in multiple types of cancer cells induce typical apoptotic cell death. This anti-apoptotic function is mediated by the FADD/c-Jun N-terminal kinase pathway. Intact $\alpha$ NAC is required for the direct binding of FADD as well as its anti-apoptosis function. Either $\alpha$ NAC depletion or the deletion of the ubiquitin-associated domain of $\alpha$ NAC sensitizes L929 cancer cells to mTRAIL treatment. Our study revealed a $\alpha$ NAC anti-apoptotic function in multiple types of cancer cells and suggested its potential in cancer therapy.

Cell Death and Disease (2014) 5, e1282; doi:10.1038/cddis.2014.192; published online 5 June 2014

Subject Category: Cancer

Extrinsic apoptosis, which is triggered by the extracellular signals that activate the death receptor (DR) family, is distinguished from intrinsic apoptosis, which is induced by intracellular signals, such as DNA damage, oxidative stress, and nutrient deprivation. ${ }^{1}$ Extrinsic apoptosis is initiated by the binding of specific ligands, such as tumor necrosis factor $\alpha(\mathrm{TNF} \alpha)$, Fas ligand, and TNF-related apoptosis-inducing ligand (TRAIL), to their corresponding receptors called DRs. ${ }^{2}$ FADD is the major adaptor transmitting apoptotic signals mediated by all known DRs. ${ }^{3}$

Following the interaction of DRs with cell death ligands and FADD, two cell death pathways are initiated by two different protein groups. The first group includes CD95/Fas, DR4/ TRAIL-R1, and DR5/TRAIL-R2, all of which recruit the death-inducing signaling complex composed of FADD and procaspase-8. ${ }^{4}$ Death-inducing signaling complex facilitates auto-proteolytic cleavage of procaspase- 8 and -10 , which confers enzymatic activity on the proteins and allows their release. ${ }^{3}$ Activation of caspase- 8 and -10 leads to proteolytic stimulation of the downstream effectors caspase-3, -6, and -7 , which can cleave intracellular substrates, such as lamin $A$,
poly-(ADP-ribose) polymerase (PARP), and MEKK1., ${ }^{4,5}$ The second group of DRs, including TNFR1, DR3, DR6, and EDAR, recruit TRADD as an adaptor protein that links DRs to TNF receptor-associated factors 2,5, receptor-interacting protein kinase (RIP1 or RIPK1), and cellular inhibitor of apoptosis, forming two signaling complexes called 'complex l' and 'complex II,' both of which can initiate apoptosis. ${ }^{2,6-8}$

JNK (c-Jun N-terminal kinases) pathway activation has been reported to mediate DR-induced apoptosis in multiple types of cell lines. ${ }^{9-13}$ DR/FADD activates JNK by two different pathways. Activated caspase is able to cleave MEKK1 and enhance its kinase activity, further inducing JNK activation via the MAPK (mitogen-activated protein kinases) cascade. ${ }^{5}$ DR/FADD is also responsible for the recruitment and activation of the kinase ASK1. ASK1 in turn activates the MAPK cascade, resulting in JNK activation. ${ }^{10,14}$ Several downstream targets, such as Bim, ${ }^{15,16} \mathrm{Bad}^{17}{ }^{17} \mathrm{p} 53^{18}$ and C-Jun, ${ }^{19}$ have been well documented to mediate the apoptotic effect of JNK activation.

NAC (nascent-polypeptide-associated complex) is a hetero-dimeric complex that was identified in higher

\footnotetext{
${ }^{1}$ Department of Dermatology, Xiangya Hospital, Central South University, Changsha, People's Republic of China; ${ }^{2}$ Department of Plastic Surgery, Xiangya Hospital, Central South University, Changsha, People's Republic of China and ${ }^{3}$ Department of Genetics and Complex Diseases, Harvard School of Public Health, Boston, MA, USA

${ }^{*}$ Corresponding author: X Chen, Department of Dermatology, Xiangya Hospital, Central South University, 87 Xiangya Road, Changsha 410008, People's Republic of China. Tel: + 867318432 7128; Fax: + 867318432 8478, E-mail: chenxiangck@gmail.com

or Z Yuan, Department of Genetics and Complex Diseases, Harvard School of Public Health, Boston, MA 02115, USA. Tel: + $6174322139 ;$ Fax: + 617 432 0107; E-mail: zyuan@hsph.harvard.edu

${ }^{4}$ These authors contributed equally to this work.

Keywords: $\alpha$ NAC; FADD; extrinsic apoptotic pathway; JNK pathway

Abbreviations: DR, death receptor; TNF, tumor necrosis factor; TRAIL, TNF-related apoptosis-inducing ligand; PRAP, poly-(ADP-ribose) polymerase; $\alpha$ NAC, nascent polypeptide-associated complex $\alpha$; m $\alpha$ NAC, mouse $\alpha$ NAC; UBA, ubiquitin-associated domain

Received 23.12.13; revised 30.3.14; accepted 31.3.14; Edited by A Stephanou
} 
eukaryotes as a ribosome-associated factor in close proximity to nascent chains. ${ }^{20,21}$ NAC containing $\alpha$ and $\beta$ subunits interacts with unfolded polypeptide chains independent of their amino-acid sequence. ${ }^{21}$ Recent studies indicate that $\alpha$ NAC has $\beta$-subunit-independent functions in development, ${ }^{22}$ transcriptional factor regulation, ${ }^{23}$ endoplasmic reticulum stress, and apoptosis. ${ }^{24}$ Clinical studies have observed elevated $\alpha$ NAC protein levels in multiple types of cancers, such as malignant gliomas, ${ }^{25}$ breast adenocarcinoma, ${ }^{26}$ and juvenile myelomonocytic leukemia. ${ }^{27}$

The functional mechanism of $\alpha \mathrm{NAC}$ in cancer progression remains largely unknown. A previous study reported that $\alpha$ NAC interacts with FADD. ${ }^{28}$ In the present study, $\alpha$ NAC was documented to exhibit an anti-apoptotic function in different cancer cell lines. Furthermore, we demonstrated that the FADD/JNK pathway is the downstream pathway of $\alpha$ NAC and that $\alpha \mathrm{NAC}$ is required for oncogenic transformation in normal fibroblast cells.

\section{Results}

Depletion of $\alpha$ NAC decreases cancer cell viability. We generated shRNAs to deplete $\alpha$ NAC (Figure 1a) in several cancer cell lines, including PC3 (prostate), H1299 (lung), MCF7 (breast), HCT116 (colon), and Hela (cervix; Figure 1b, Supplementary Figure 1). Surprisingly, aNAC was able to decrease cell viability by over $75 \%$ in all cancer cell lines except Hela cells (Figure 3a). To avoid the off-target effect of shRNA, we rescued $\alpha$ NAC protein levels in $\alpha$ NAC-depleted PC3 cells using mouse $\alpha$ NAC (m $\alpha$ NAC; Figure 1c). m $\alpha$ NAC, which exhibits $99 \%$ identity with human $\alpha \mathrm{NAC}$ (Supplementary Figure 2), was able to recover over $60 \%$ of cell viability (Figure 1d). Cell growth curve analyses were performed. $\alpha$ NAC-depleted cells grew slower than control cells, whereas the introduction of exogenous m $\alpha$ NAC into $\alpha$ NAC-depleted cells was able to rescue the growth defect (Figure 1e).

\section{Depletion of $\alpha$ NAC induces caspase-8-dependent apoptosis.} To pursue the mechanism of $\alpha$ NAC depletion-mediated cell viability inhibition, Annexin $\mathrm{V}$ assays were performed. Less than $5 \%$ of control PC3 cells exhibited Annexin V-positive signals, whereas approximately $90 \%$ of $\alpha$ NAC-depleted cells exhibited Annexin V-positive signals (Figure 2a). To verify the results of the Annexin $V$ assay, several apoptotic molecular markers were investigated. The levels of caspase-3, -8, and -9 cleavage were much higher in $\alpha$ NACdepleted PC3 cells compared with the control. PARP, which is a substrate of caspases, was cleaved more frequently in $\alpha$ NAC-depleted cells than in control cells (Figure $2 b$ ). Caspase-3 receives signals from caspase-8 or -9 in response to the extrinsic apoptotic pathway or the intrinsic apoptotic pathway, respectively. Thus, we introduced dominant-negative caspase-3, -8, or -9 into control or $\alpha$ NACdepleted cells and observed caspase- 3 activity. In PC3 cells transfected with control empty vector, caspase-3 activity increased twofold. In caspase-3 DN- and caspase-8 DNtransfected cells, caspase-3 activity was inhibited. Interestingly, caspase-9 DN was not able to inhibit caspase-3 activity efficiently (Figure 2c). This result suggests that the apoptotic process was initiated by the extrinsic pathway when $\alpha$ NAC was depleted.

FADD mediates apoptosis under $\alpha$ NAC-depleted conditions. Among the cancer cells we detected, HeLa cells exhibited a much lower cell death response under $\alpha$ NAC depletion compared with the other cells we measured. Over $50 \%$ of HeLa cells survived $\alpha$ NAC depletion (Figure 3a). HeLa cells harbors human papillomavirus 18 virus, which express the E6 protein that is able to inhibit $p 53^{29}$ and $F A D D^{30}$ function and protect cells from apoptosis. PC3 and PC3-E6 cells were used to test the hypothesis that E6 was responsible for HeLa cell resistance. Less than $5 \%$ of PC3 sh $\alpha$ NAC cells survived, whereas over $80 \%$ of PC3-E6 sh $\alpha$ NAC cells survived (Figure 3b). $\alpha$ NAC depletion efficiently induced cell death in PC3 and H1299 cells, both of which cells are functional p53null. Therefore, we further investigated the role of FADD role. When FADD was co-depleted with $\alpha$ NAC, approximately $80 \%$ of the cells survived (Figure $3 c$ ). Caspase- 3 , -8 , and -9 activities were measured in the indicated cells (Figure $3 d$ ). The activities of all three caspases were enhanced only in $\alpha$ NAC-depleted cells and inhibited in FADD-depleted cells. PARP and caspase-3 cleavage were decreased in the 'sh $\alpha \mathrm{NAC}+$ shFADD' group as well (Figure 3e). The statistic analysis for p-JNK signal was done (Supplementary Figure 5).

The MAPK/JNK pathway mediates the apoptotic effect of aNAC depletion. To investigate the signal transduction under $\alpha$ NAC depletion conditions, Cancer 10-pathway assays were performed. The activity of two pathways, MAPK/ERK and MAPK/JNK, increased over twofold. In particular, the JNK pathway increased over fivefold (Figure 4a). To block MEKK and JNK activity, two dominant-negative kinase-dead proteins, MEKK-CF-KR and JNK-APF, were introduced into $\alpha$ NAC-depleted or control PC3 cells. Both kinase-dead proteins were able to inhibit JNK phosphorylation and caspase-3 cleavage, and JNK-APF was more potent than MEKK-CF-KR (Figure 4b). The statistic analysis for p-JNK signal was done (Supplementary Figure 6). Colony formation experiments revealed that both kinase-dead proteins were able to rescue the cell viability that was decreased by $\alpha$ NAC depletion and that JNK-APF was more potent than MEKK-CFKR (Figure 4c). Pathway activities were measured using a transcriptional luciferase reporter assay. JNK-APF was able to inhibit AP1 transcriptional activity over $70 \%$. MEKK-CF-KR was able to inhibit SRE transcriptional activity over $50 \%$ (Supplementary Figure 3).

Intact $\alpha$ NAC protein is necessary for anti-apoptotic function. $\alpha$ NAC protein harbors three functional regions related to DNA binding, C-Jun binding, or ubiquitin. Based on the functional regions, we constructed a series of deletion mutants of $\alpha$ NAC (Figure 5a). The mutants were introduced into $\alpha$ NAC-depleted or control PC3 cells to rescue the apoptotic phenotype. Colony formation experiments revealed that only intact m $\alpha \mathrm{NAC}$ was able to rescue cell viability efficiently (Supplementary Figure 4, Figure 5b). Correspondingly, JNK pathway activity could only be inhibited by intact m $\alpha$ NAC under $\alpha$ NAC depletion conditions (Figure $5 \mathrm{c}$ ). 

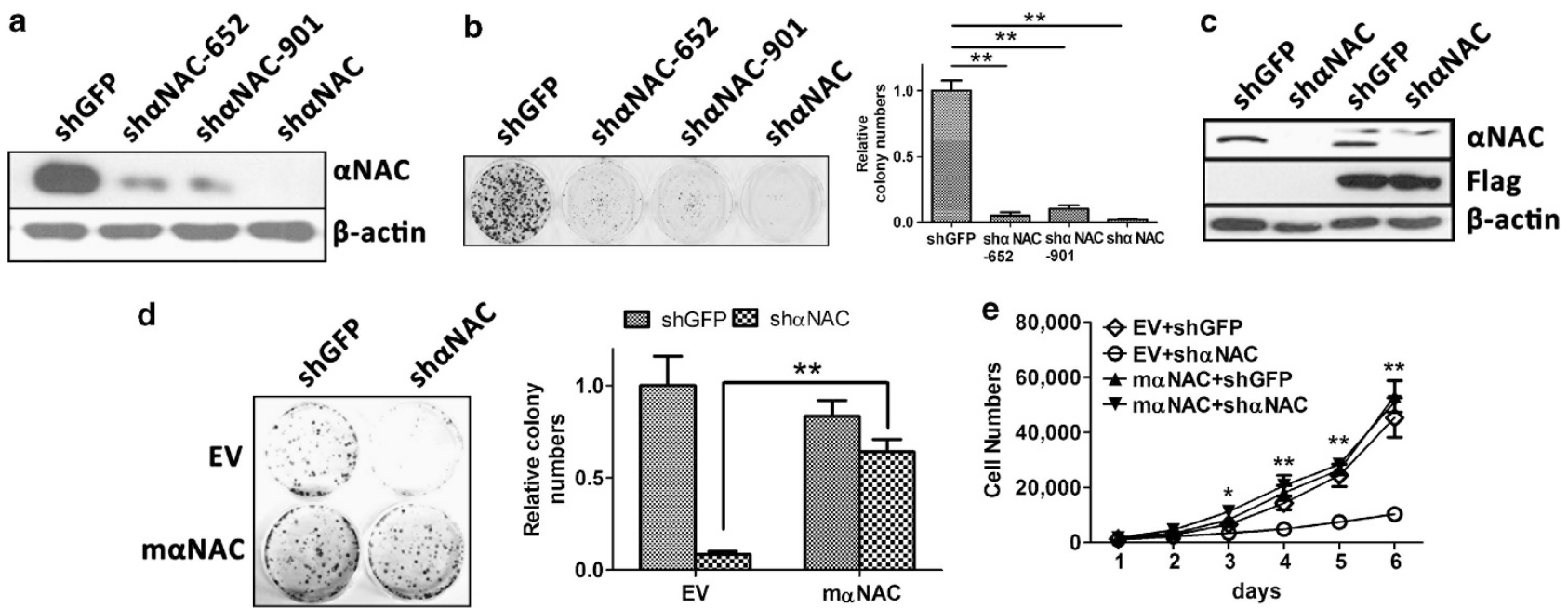

Figure 1 Depletion of $\alpha$ NAC decreased cell viability. (a) Two target sequences (652 and 901) were designed against $\alpha$ NAC at different sites. These two small hairpin RNAs (shRNA) were introduced into PC3 cells by lentiviral transduction. After selection, cells were harvested and subjected to western blot analysis. Cells (sh $\alpha$ NAC) infected with mixed lentiviral mixtures achieved better depletion efficiency than each alone. (b) $\alpha$ NAC were depleted in PC3 cells by two independent sequences. Cells were selected by puromycin for 2 days and subjected to colony formation assay. (c) Western blots were performed to verify the maNAC recovery with or without endogenous $\alpha$ NAC. (d) $\mathrm{m} \alpha \mathrm{NAC}$ rescued cell death induced by $\alpha \mathrm{NAC}$ depletion. Colony formation assays were performed to investigate the viability of the indicated PC3 cell (left panel, EV means empty vector). Colonies were counted using ImageJ software (right panel). (e) maNAC reverses cell growth inhibition induced by $\alpha$ NAC depletion. The indicated cells were seeded into 96-well plates at 1000 cells/well. Cells were trypsinized and counted under a microscope every day. Triplicate samples were made for each condition

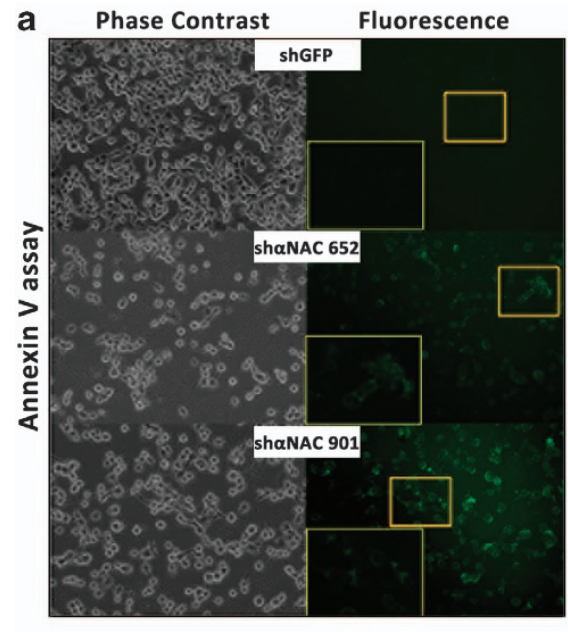

b
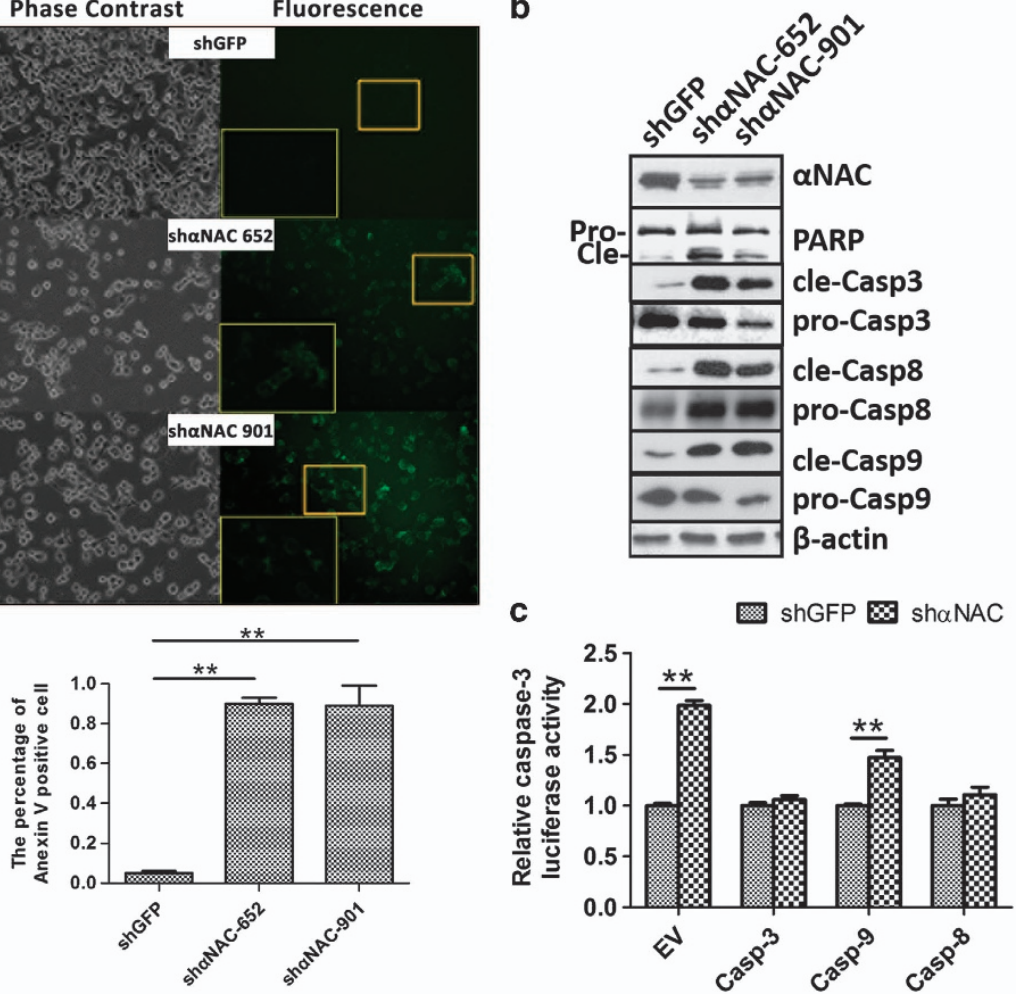

Figure 2 Depletion of $\alpha$ NAC induces apoptotic cell death and casp8-dependent casp3 activation. (a) Annexin V assay. PC3 cells depleted with $\alpha$ NAC exhibit positive fluorescent signals (upper panel). We counted 500 cells in each group and performed statistical analysis (lower panel). (b) The indicated PC3 cells were harvested and subjected for western blot analysis. The indicated antibodies were used to probe the membrane. (c) PC3 cells were transfected with empty vector or plasmids expressing caspase $-3,-8$, or -9 DN. After $12 \mathrm{~h}$, the medium was switched to lentiviral supernatant. After $6 \mathrm{~h}$, the medium was changed to fresh media. After $48 \mathrm{~h}$, cells were harvested and subjected to caspase-3 enzyme activity assay

Interestingly, the overexpression of the D180-215 deletion mutant, which is an ubiquitin-associated domain (UBA domain), in PC3 cells decreased viability over $60 \%$, and AP-
1 activity increased correspondingly. Cleaved caspase-3 was probed. Only intact m $\alpha$ NAC was able to inhibit caspase-3 cleavage completely (Figure $5 d$ ). 
a
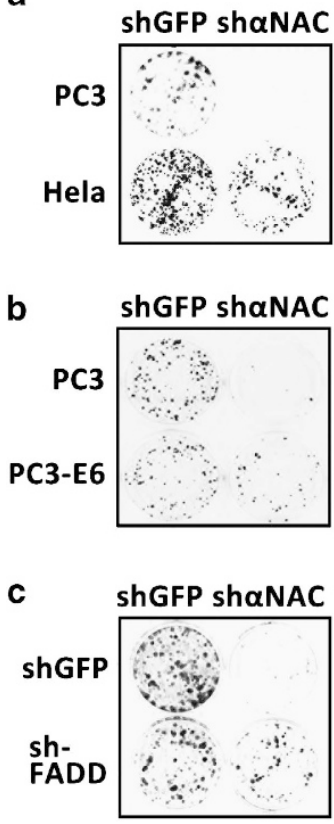
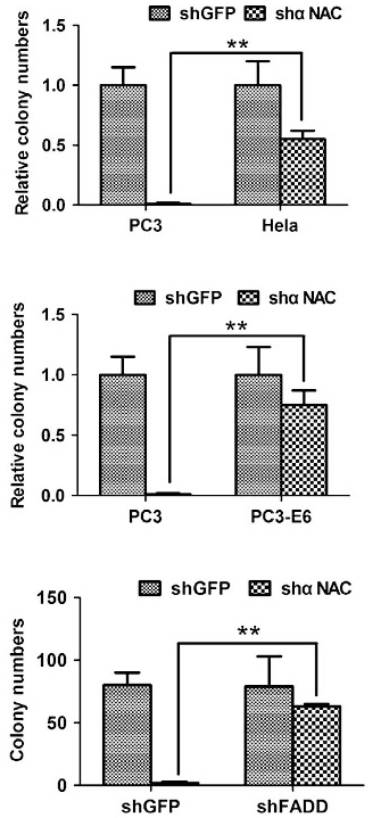
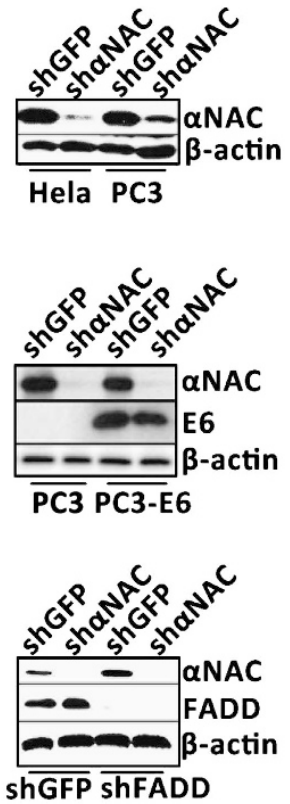

d shGFP+shGFP $\otimes$ shGFP+sha NAC 目 shFADD+shGFP 四 shFADD+sha NAC

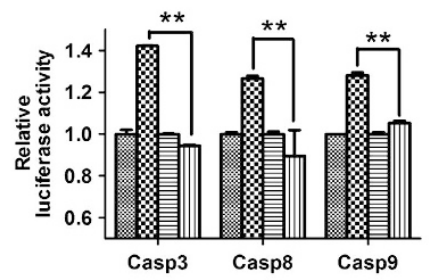

e

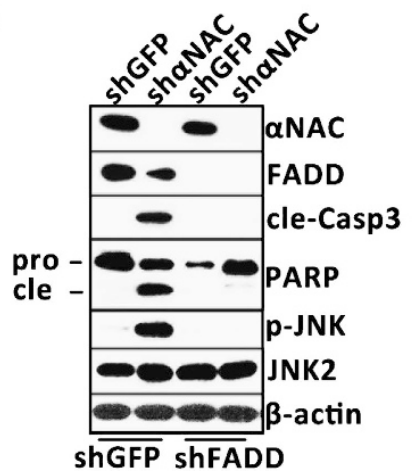

Figure 3 FADD mediates the apoptotic effect of $\alpha$ NAC depletion. (a) With or without $\alpha$ NAC depletion, PC 3 cells or Hela cells were subjected to colony formation assays (left panel). (b) With or without $\alpha$ NAC depletion, PC3 or PC3-E6 cells were subjected to colony formation assay (left panel). (c) PC3 cells with $\alpha$ NAC and (or) FADD depletion were subjected to colony formation assays (left panel). For $a, b$ and $c$, all colonies were counted and analyzed (middle panel). When the cells were seeded for colony formation assays, $1 \times 10^{6}$ cells were kept for western blots to confirm the depletion efficiency (right panel). Some cells from experiment c were kept for caspase $-3,-8$ and -9 activity assays (Figure $3 \mathrm{~d}$ ) or western blots (Figure $3 \mathrm{e}$ )

a
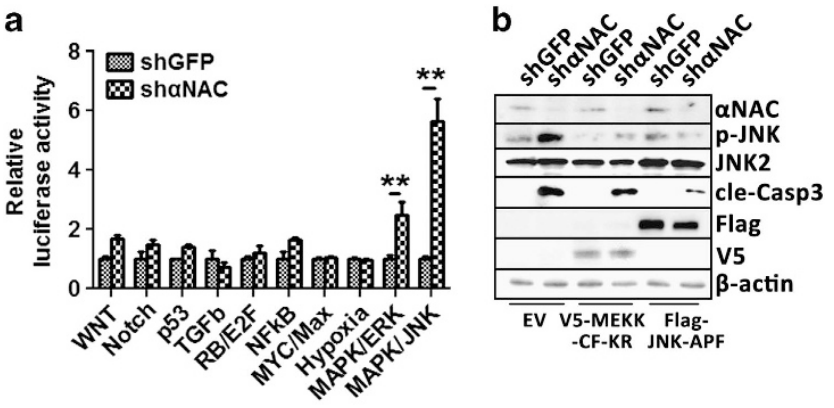

EV V5-MEKK Flag-CF-KR JNK-APF

C
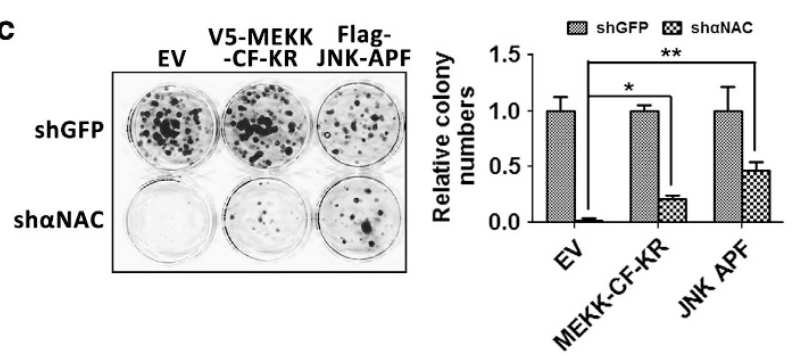

Figure 4 The MAPK/JNK pathway partially mediates the apoptotic effect of $\alpha$ NAC depletion. (a) PC3 shGFP or sh $\alpha$ NAC cells were subjected to Cancer 10-pathway reporter arrays. Luciferase activity represents the pathway activity. For b and c, empty vector, MEKK-KR, or JNK-APF was transfected into PC3 cells. After selection, $\alpha$ NAC was depleted in all three cell lines. Cells were harvested for western blot analysis to investigate the levels of the indicated proteins (b) or were subjected to colony formation assays (c, left panel). Colonies were counted and analyzed (c, right panel)

The middle and C-terminal regions of $\alpha \mathrm{NAC}$ and the $\mathrm{N}$-terminus of FADD are necessary for interaction. Endogenous protein interactions between $\alpha$ NAC and FADD were investigated. A FADD antibody was able to pull down $\alpha$ NAC protein (Figure 6a). Immunofluorescence (IF) revealed co-localization between endogenous $\alpha$ NAC and FADD protein (Figure 6b). To investigate the interaction region in both proteins, immunoprecipitation (IP) was performed in a series of $\mathrm{m} \alpha \mathrm{NAC}$ or FADD deletion mutants. Flag-tagged full-length maNAC successfully immunoprecipitated FADD protein. When 89-128 amino acids (aa) or 180-215 aa of m $\alpha$ NAC were deleted, the interaction was interrupted (Figure $6 \mathrm{c}$ ). Flag-tagged m $\alpha$ NAC was able to precipitate the FADD fulllength protein and mutants, with the exception of the 1-153 aa deletion mutant (Figure 6d).

Depletion of m $\alpha$ NAC or the addition of the D185-215 mutant sensitized the murine aneuploid fibrosarcoma cell line L929 to mTRAIL treatment. TRAIL has been used clinically for tumor therapy purposes to induce extrinsic apoptotic cell death. We used L929 cells, which are sensitive to mTRAIL treatment in vitro, as a model. mTRAIL alone induced $45 \%$ cell death. The combination of mTRAIL with the D185-215 mutant or shNAC induced more cell death. Interestingly, the D180-215 mutant only induced approximately $40 \%$ cell death, whereas $85 \%$ cell death was observed when the mutant was combined with mTRAIL treatment (Figures $7 a$ and $b$ ).

\section{Discussion}

The extrinsic apoptotic pathway has a very important role in cancer progression as well as in cancer therapy. Malignant cells develop several strategies to inhibit the extrinsic apoptotic pathway activity against death signals, such as TNF $\alpha$, TRAIL, and Fas ligand. Downregulation of DRs is often 


\section{a}

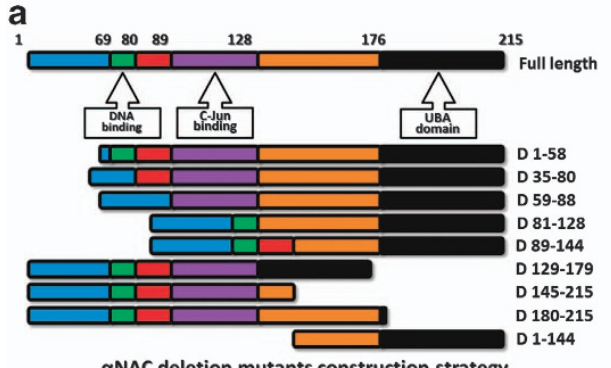

aNAC deletion mutants construction strategy

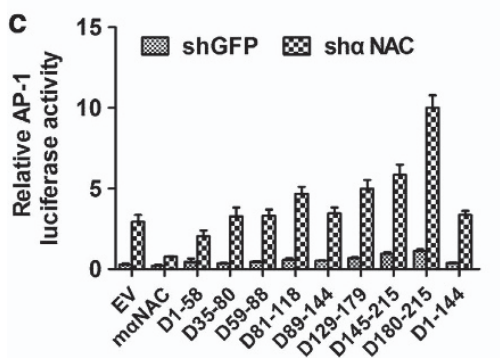

d 4 b

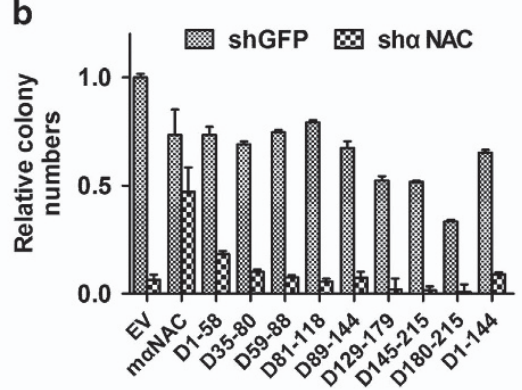

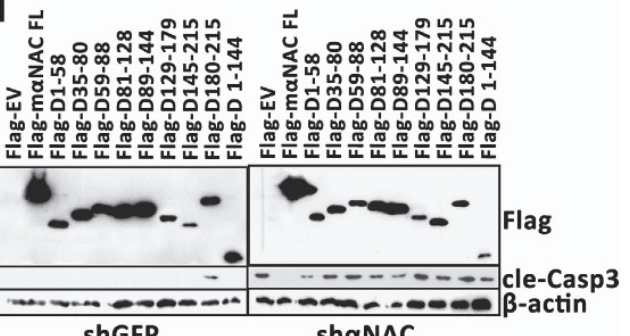

Figure 5 Intact $\alpha$ NAC is required for anti-apoptotic function. (a) The strategy for creating the $\alpha$ NAC deletion mutant. All mutants were introduced into PC3 cells to make stable cells, and the cells were then infected with shGFP or sh $\alpha$ NAC lentiviruses. All cells were subjected to colony formation assays and counted the colony numbers (b), JNK pathway reporter assays (c), and western blot analysis (d)

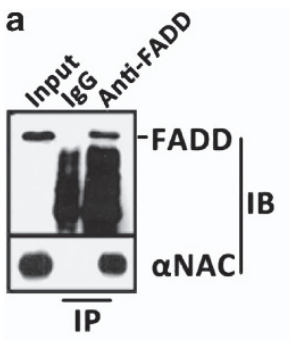

C

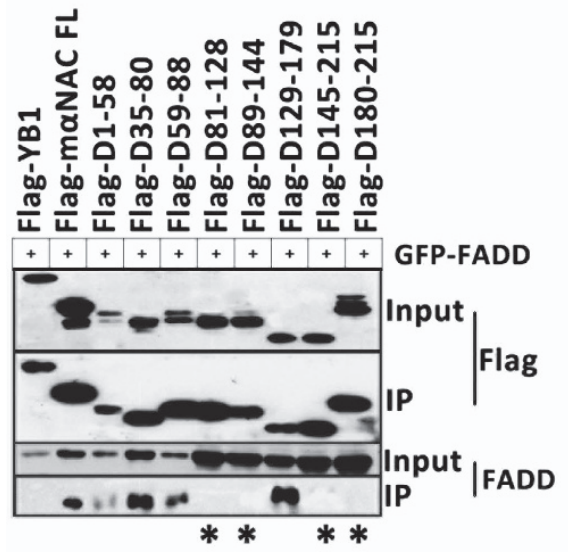

b

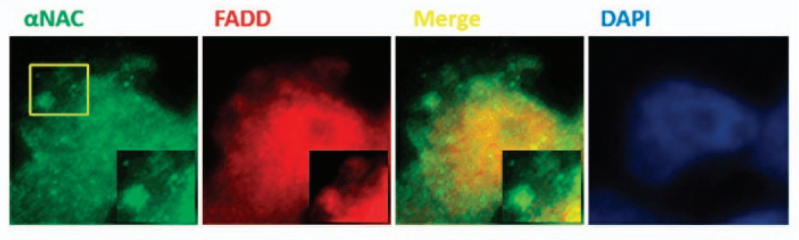

d

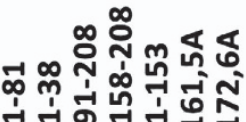

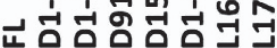

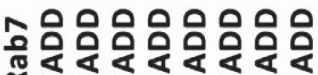

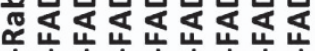

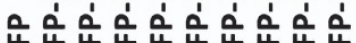

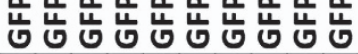

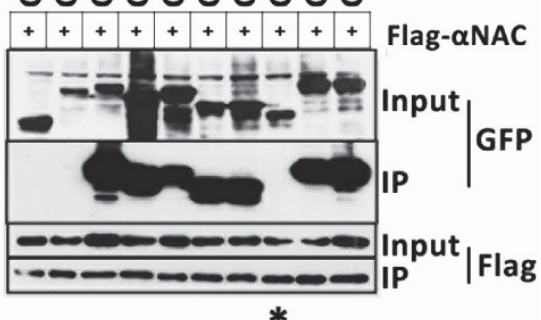

Figure 6 Identification of the interaction domain of $\alpha$ NAC and FADD. (a) Approximately $2 \times 10^{7}$ cells were harvested, lysed in RIPA buffer, and separated into three parts. Approximately $5 \%$ lysate was used as an 'input' sample, half of the remaining lysate was incubated with normal rabbit lgG, which was severed as negative control, and the other half of the remaining lysate was incubated with FADD antibody. After overnight incubation at $4{ }^{\circ} \mathrm{C}$, the samples were washed three times with PBS and then subjected to western blot analysis. (b) The Endogenous $\alpha$ NAC and FADD distribution were investigated by IF. (c) Full-length GFP-tagged FADD was co-transfected with full-length m $\alpha$ NAC or deletion mutants. IP was performed with M2 beads to precipitate flag-tagged maNAC. Co-precipitated FADD was probed using an anti-FADD antibody. An un-related protein, YB1, served as a negative control. (d) Full-length flag-tagged maNAC was co-transfected with full-length GFP-tagged FADD or deletion mutants. IP was performed to pull down M2 beads to precipitate flag-tagged maNAC. Co-precipitated FADD was probed using an anti-FADD antibody. An un-related protein, Rab7, served as a negative control 
a
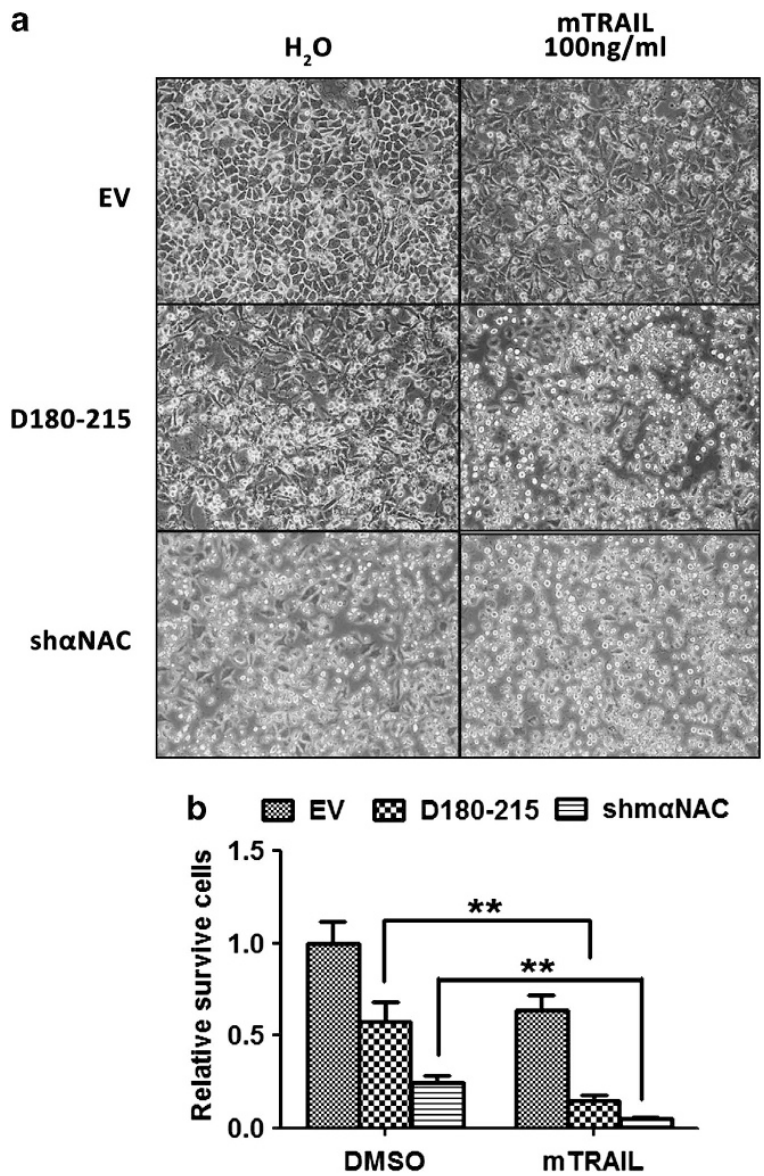

Figure 7 Depletion of $\alpha$ NAC or the expression of the D180-215 mutant sensitizes L929 cells to mTRAIL. L929 cells were infected with virus to deplete $m \propto N A C$ or to express the D180-215 mutant. After selection, cells were seeded into six-well plates and treated with mouse TRAIL. After $6 \mathrm{~h}$, cells were imaged (a) and trypsinized for trypan blue assay (b)

associated with resistance to drug-induced cell death. ${ }^{31-33}$ Downregulation of Fas signaling can be attributed to changes at the genomic, transcriptional, and posttranslational levels. Mutations in the death domain of Fas have been reported in $7 \%$ of metastatic melanomas, ${ }^{34}$ resulting in an abnormal protein that exhibits abrogated associations with FADD and procaspase-8. A more common event is silencing of the Fas receptor promoter. ${ }^{35-37}$ Another means of attenuating Fas expression is its selective inhibition from being expressed on the cell surface. ${ }^{38,39}$ All of those pathological changes are able to attenuate signal transduction and cellular responses to death signals. Recent studies have revealed that targeting several downstream proteins, such as cellular FLICE-like inhibitory protein ${ }^{40,41}$ and caspase- $8,{ }^{42}$ is able to overcome the drug resistance caused by the downregulation of DRs. Our study provides evidence that the depletion of $\alpha$ NAC potently induces apoptotic cell death in multiple cancer cell lines but not in normal fibroblast cells. $\alpha$ NAC is a potential candidate for cancer therapy.

aNAC harbors a UBA domain in its extreme C-terminus. The UBA domain is a small structural domain in proteins that is characterized by a three-helix bundle and is typically associated with the ubiquitin pathway. Binding of the long-chain ubiquitin of the proteins to UBA proteins is able to promote their degradation. ${ }^{43}$ Some proteins harboring UBA domains have been identified in the ubiquitin-associated proteasome pathway, such as nuclear factor- $\kappa B$ essential modulator, R23A, Dsk2, and NBR1. ${ }^{44-47}$ All of these proteins belong to class 1,2 , or 4 UBAs. Class 3 , to which $\alpha$ NAC belongs, exhibits no demonstrable binding affinity with longchain ubiquitin in yeast (Egd2 is homologue of $\alpha N A C$ in yeast). We do not find any evidence to support that $\alpha$ NAC regulates FADD by regulating protein stability because the depletion or overexpression of $\alpha \mathrm{NAC}$ did not significantly change the FADD level. Therefore, it would be interesting to investigate the binding affinity between FADD and caspase-8 or RIP1 with or without $\alpha$ NAC.

Interestingly, the D180-215 mutant, deleted with UBA domain, activates AP1 pathway and inhibits cell viability when overexpressed in PC3 cells only. D180-215 mutant also sensitized mTRAIL-induced L929 cell apoptosis in vitro. Considering those effect is consistent with $\alpha$ NAC depletion, we propose UBA domain has an important role in $\alpha$ NAC antiapoptotic function. Further investigation focusing on this domain would be helpful to clarify the mechanism.

Depletion of FADD completely blocked JNK phosphorylation (Figure 3e), suggesting that JNK activation is the downstream of FADD. Depletion of FADD efficiently recovers cell apoptosis induced by $\alpha \mathrm{NAC}$ depletion. Overexpression of JNK APF in $\alpha$ NAC-depleted cells was able to recover cell viability to $40 \%$ (Figure 4c), although it blocked endogenous JNK activity over 70\% (Supplementary Figure 3). Therefore, the JNK pathway is the major but not the only pathway to mediate the $\alpha$ NAC/FADD effect. The signal transduction from FADD to JNK has two potential pathways, correspondingly mediated by MEKK $1^{4}$ or ASK. ${ }^{14}$ When endogenous MEKK1 was inhibited by MEKK-CF-KR, cell viability decreased to approximately $20 \%$. Thus, MEKK1 is not the only mediator of signal transduction from FADD to JNK (Supplementary Figure 5).

Caspases response to apoptotic signal by two ways. For a short-time stimulation, caspases were cleaved and activated. For sustained stress, JNK and other pathways promote caspase gene transcription and elevate those protein levels. ${ }^{48}$ In this study, we used lentivirus to introduce siRNA against aNAC into cells. It takes about 3 days. We found not only caspase cleavage but also caspase protein level elevation in $\alpha$ NAC-depleted cells. It is consistent with the JNK activation we found.

When cell undergo extrinsic apoptosis, in so-called type 1 cells, proteolytic activation of caspases-3 by caspase- 8 suffices for efficient apoptosis induction. In so-called type 2 cells, killing requires amplification of the caspase cascade. This can be achieved through caspase-8-mediated proteolytic activation of the pro-apoptotic Bcl-2 homology domain $(\mathrm{BH})$ 3-only protein $\mathrm{BH} 3$-interacting domain death agonist (Bid), which then causes mitochondrial outer membrane permeabilization. ${ }^{49}$ Further investigation is required to clarify BID's role when $\alpha$ NAC was depleted.

Our study revealed that $\alpha$ NAC, a nascent peptide-associated protein, exhibits an anti-apoptotic function independent of the NAC complex in cancer cells. The anti-apoptotic mechanism of $\alpha \mathrm{NAC}$ was concluded as a diagram 
(Figure 8). $\alpha \mathrm{NAC}$ is a potential therapy target, and further study on the mechanism of $\alpha$ NAC regulation of FADD is necessary.

\section{Materials and Methods}

PCR and cloning. Oligonucleotides were synthesized as per protocol by Invitrogen (Grand Island, NY, USA) and are listed in Supplementary Table 1. C-MYC, ${ }^{50}$ myr-AKT, ${ }^{51}$ HRasV12 (Cat. 1768), MEKK1-CF-KR, ${ }^{52}$ JNK-APF $^{53}$ caspase-3 DN (dominant negative), ${ }^{54}$ caspase-8 DN, ${ }^{55}$ and caspase-9 DN ${ }^{55}$ were purchased from Addgene (http://www.addgene.org) and were sub-cloned into corresponding lentiviral expression plasmids. All the details of those plasmids are available in the references correspondingly. PCRs were performed with KOD Taq polymerase (TOYOBO, Novi, MI, USA) and Mastercycler nexus (Eppendorf, Hamburg, Germany).

Cell culture, transfection, and reagents. PC3, MCF7, H1299, MDA-MB-231, U2OS, and 293T cells were purchased from the American Type Culture Collection and were maintained in their corresponding media as standard protocol. PC3-E6 cells were created and maintained following the published MCF7-E6 building methods. ${ }^{56}$ All cell culture reagents were purchased from Gibco (New York, NY, USA). Mouse TRAIL (Cat. SRP3237) and other reagents were purchased from Sigma (St. Louis, MO, USA) unless otherwise indicated. Transfections were performed with Lipofectamine 2000 (Invitrogen) according to the standard instructions. In each experiment, the amounts of the transfected plasmids were consistent, and an empty vector was used to compensate for any remaining amount. Each experiment was repeated three times.

Cancer 10-pathway reporter arrays. The Cancer 10-pathway Reporter kit (CCA-001L) was purchased from SABiosciences (Frederick, MA, USA). The screening process was performed following the standard manual. In brief, MCF7 cells were seeded into 96-well plates at a density of $1 \times 10^{4}$ cells per well. Reverse transfections were performed when the cells were seeded. After $48 \mathrm{~h}$, the cells were harvested and subjected to dual luciferase analysis, which was performed using the Dual-Luciferase Reporter Assay System (E1960, Promega, Madison, WI, USA).

Western blot analysis, IP, IF, and antibodies. Western blot analysis, IPs, and IF were performed according to the described procedures. ${ }^{50} \alpha \mathrm{NAC}$ (sc-87324, 1:500), BCL2 (sc-492, 1:1000), MYC (SC-40, 1:2000), HRas (sc-34, 1:500), JNK2 (sc-827, 1:1000), FADD (sc-5559, 1:500), HPV16 E6 (sc-1584, 1:1000), GFP (sc-390394, 1:2000), and V5-Probe (sc-81594, 1:500) antibodies and Protein A/G PLUS agarose beads (SC-2003) were purchased from Santa

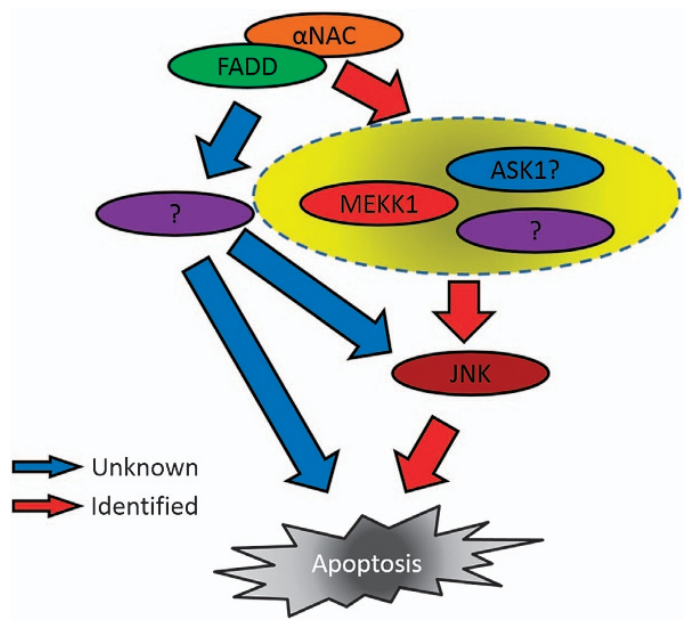

Figure 8 Schematic diagram for cell apoptosis induced by $\alpha$ NAC depletion. FADD exclusively mediates $\alpha$ NAC anti-apoptotic effect. The one of the downstream pathway of FADD is JNK pathway. MEKK1, ASK1, and (or) other kinases transduce the signal from FADD to JNK. There is another (or others) pathway partially mediated the apoptotic effect of $\alpha$ NAC depletion in JNK-independent manner
Cruz Biotechnology (Dallas, TX, USA). Flag (F4042, 1:5000, mouse), Flag (F2555, 1:1000, rabbit), and actin (A4700, 1:5000) antibodies and Anti-FLAG M2 Magnetic Beads (M8823) were purchased from Sigma. PARP (9532, 1:500), cleaved caspase-3 (9664, 1:500), cleaved caspase-8 $(9496,1: 500)$, cleaved caspase-9 (9505, 1:500), and p-JNK $(4668,1: 1000)$ antibodies were purchased from Cell Signaling Technology (Danvers, MA, USA). The goat anti-mouse (SC-2005, 1:500) and goat anti-rabbit (SC-2004, 1:500) secondary antibodies were purchased from Santa Cruz Biotechnology. The secondary antibodies used for IF were purchased from Invitrogen, including Alexa Fluor 594 (A11020, 1:500) and Alexa Fluor 488 (A11034, 1:500). The blot images were taken using a ChemiDoc XRS + system (Bio-Rad, Hercules, CA, USA). IF images were taken by microscopy (CX4132RFL, OLYMPUS). IP was performed following a previous protocol. ${ }^{52}$

Virus packaging and infection. The pLKO1 plasmid, pLenti entrydestination system, ${ }^{57}$ and helper vectors, VSVG and delta8.2, were purchased from Addgene. Virus packaging and target cell infection were performed following standard protocols from Addgene. Stable cell lines were created by selection with $1 \mu \mathrm{g} / \mathrm{ml}$ puromycin (P9620, Sigma) for 6 days.

Annexin V assay. The Annexin V-FITC Apoptosis Detection Kit was purchased from Sigma (APOAF-50TST). Cells were seeded on sterile cover slides in $35 \mathrm{~mm}$ dishes. The following day, cells were washed with PBS twice and incubated with the Annexin V-FITC working solution for $30 \mathrm{~min}$. Slides were placed cell-side down on slides, and positively stained cells were counted under a microscope.

Caspase enzyme activity assay. Three caspase activity assay kits, caspase-Glo3/7 (G8090), caspase-Glo8 (G8200), and caspase-Glo9 (G8210), were purchased from Promega. Experiments were performed according to the manufacturer's manual.

Colony formation assay. Cells were trypsinized and suspended in culture medium. After counting, cells were diluted to $50 \mathrm{cell} / \mathrm{s} / \mathrm{ml}$. Approximately $2 \mathrm{ml}$ of cell suspension (approximately 100 cells) was seeded into each $60 \mathrm{~mm}$ dish. The medium was changed every 1 or 2 days. After 2 weeks under normal conditions, cells were fixed in $4 \%$ formaldehyde for $10 \mathrm{~min}$. After two washes with PBS, $0.05 \%$ crystal violet was added to the dishes. After $15 \mathrm{~min}$, crystal violet was removed, and the cells were washed twice with PBS (upper panel). Colonies were counted using ImageJ software (version 1.43; http://rsbweb.nih.gov/ij/download.html).

Statistical analysis and software. All experiments were repeated three times. For each histogram, the average value and error bar were calculated from three independent experiments. GraphPad Prism 5 was used to analyze the data and create histograms. One-way ANOVA was used to assess statistical significance for certain data. A double asterisk $\left.{ }^{* *}\right)$ indicates $P<0.01$, and a single asterisk $\left(^{*}\right)$ indicates $P<0.05$.

\section{Conflict of Interest}

The authors declare no conflict of interest.

Acknowledgements. This work was supported by grant 81201240 from National Natural Science Foundation of China (W Zeng), National Natural Science Foundation of China 81225013 for Distinguished Young Scholar (X Chen), and grant 81301986 from National Natural Science Foundation of China (M Qi).

1. Kroemer G, Galluzzi L, Brenner C. Mitochondrial membrane permeabilization in cell death. Physiol Rev 2007; 87: 99-163.

2. Mahmood Z, Shukla Y. Death receptors: targets for cancer therapy. Exp Cell Res 2010; 316: 887-899.

3. Tourneur L, Chiocchia G. FADD: a regulator of life and death. Trends Immunol 2010; 31 : 260-269.

4. Slee EA, Adrain C, Martin SJ. Executioner caspase-3, -6, and -7 perform distinct, nonredundant roles during the demolition phase of apoptosis. J Biol Chem 2001; 276: 7320-7326.

5. Widmann C, Gerwins P, Johnson NL, Jarpe MB, Johnson GL. MEK kinase 1, a substrate for DEVD-directed caspases, is involved in genotoxin-induced apoptosis. Mol Cell Biol 1998; 18: 2416-2429.

6. Lavrik I, Golks A, Krammer PH. Death receptor signaling. J Cell Sci 2005; 118(Pt 2): 265-267.

7. Wilson NS, Dixit V, Ashkenazi A. Death receptor signal transducers: nodes of coordination in immune signaling networks. Nat Immunol 2009; 10: 348-355. 
8. Declercq W, Vanden Berghe T, Vandenabeele P. RIP kinases at the crossroads of cell death and survival. Cell 2009; 138: 229-232.

9. Brenner B, Koppenhoefer U, Weinstock C, Linderkamp O, Lang F, Gulbins E. Fas- or ceramide-induced apoptosis is mediated by a Rac1-regulated activation of Jun N-terminal kinase/p38 kinases and GADD153. J Biol Chem 1997; 272: 22173-22181.

10. Yang X, Khosravi-Far R, Chang HY, Baltimore D. Daxx a novel Fas-binding protein that activates JNK and apoptosis. Cell 1997; 89: 1067-1076.

11. Le-Niculescu H, Bonfoco E, Kasuya Y, Claret FX, Green DR, Karin M. Withdrawal of survival factors results in activation of the JNK pathway in neuronal cells leading to Fas ligand induction and cell death. Mol Cell Biol 1999; 19: 751-763.

12. Costa-Pereira AP, McKenna SL, Cotter TG. Activation of SAPK/JNK by camptothecin sensitizes androgen-independent prostate cancer cells to Fas-induced apoptosis. $\mathrm{Br} \mathrm{J}$ Cancer 2000; 82: 1827-1834.

13. Zhang J, Gao JX, Salojin K, Shao Q, Grattan M, Meagher $C$ et al. Regulation of fas ligand expression during activation-induced cell death in T cells by $\mathrm{p} 38$ mitogen-activated protein kinase and c-Jun NH2-terminal kinase. J Exp Med 2000; 191: 1017-1030.

14. Curtin JF, Cotter TG. Defects in death-inducing signalling complex formation prevent JNK activation and Fas-mediated apoptosis in DU 145 prostate carcinoma cells. Br J Cancer 2003; 89: 1950-1957.

15. Geissler A, Haun F, Frank DO, Wieland K, Simon MM, Idzko M et al. Apoptosis induced by the fungal pathogen gliotoxin requires a triple phosphorylation of Bim by JNK. Cell Death Differ 2013; 20: 1317-1329.

16. Putcha GV, Le S, Frank S, Besirli CG, Clark K, Chu B et al. JNK-mediated BIM phosphorylation potentiates BAX-dependent apoptosis. Neuron 2003; 38: 899-914.

17. Zhang J, Liu J, Yu C, Lin A. BAD Ser128 is not phosphorylated by c-Jun NH2-terminal kinase for promoting apoptosis. Cancer Res 2005; 65: 8372-8378.

18. Miller FD, Pozniak CD, Walsh GS. Neuronal life and death: an essential role for the p53 family. Cell Death Differ 2000; 7: 880-888.

19. Wagner EF, Nebreda AR. Signal integration by JNK and p38 MAPK pathways in cancer development. Nat Rev Cancer 2009; 9: 537-549.

20. Zhang Y, Berndt U, Golz H, Tais A, Oellerer S, Wolfle T et al. NAC functions as a modulator of SRP during the early steps of protein targeting to the endoplasmic reticulum. Mol Biol Cell 2012; 23: 3027-3040

21. Lauring B, Wang S, Sakai H, Davis TA, Wiedmann B, Kreibich G et al. Nascentpolypeptide-associated complex: a bridge between ribosome and cytosol. Cold Spring Harb Symp Quant Biol 1995; 60: 47-56.

22. Meury T, Akhouayri O, Jafarov T, Mandic V, St-Arnaud R. Nuclear alpha NAC influences bone matrix mineralization and osteoblast maturation in vivo. Mol Cell Biol 2010; 30: 43-53.

23. Yotov WV, Moreau A, St-Arnaud R. The alpha chain of the nascent polypeptide-associated complex functions as a transcriptional coactivator. Mol Cell Biol 1998; 18: 1303-1311.

24. Hotokezaka Y, van Leyen K, Lo EH, Beatrix B, Katayama I, Jin G et al. alphaNAC depletion as an initiator of ER stress-induced apoptosis in hypoxia. Cell Death Differ 2009; 16: 1505-1514.

25. Kroes RA, Jastrow A, McLone MG, Yamamoto H, Colley P, Kersey DS et al. The identification of novel therapeutic targets for the treatment of malignant brain tumors. Cancer Lett 2000; 156: 191-198.

26. Murphy JP, Pinto DM. Temporal proteomic analysis of IGF-1R signalling in MCF-7 breast adenocarcinoma cells. Proteomics 2010; 10: 1847-1860.

27. Hammerle K, Shayan P, Niemeyer CM, Flotho C. Expression analysis of alpha-NAC and ANX2 in juvenile myelomonocytic leukemia using SMART polymerase chain reaction and 'virtual Northern' hybridization. Cancer Genet Cytogenet 2003; 142: 149-152.

28. Stilo R, Liguoro D, di Jeso B, Leonardi A, Vito P. The alpha-chain of the nascent polypeptide-associated complex binds to and regulates FADD function. Biochem Biophys Res Commun 2003; 303: 1034-1041.

29. Havre PA, Yuan J, Hedrick L, Cho KR, Glazer PM. p53 inactivation by HPV16 E6 results in increased mutagenesis in human cells. Cancer Res 1995; 55: 4420-4424.

30. Garnett TO, Filippova M, Duerksen-Hughes PJ. Accelerated degradation of FADD and procaspase 8 in cells expressing human papilloma virus 16 E6 impairs TRAlL-mediated apoptosis. Cell Death Differ 2006; 13: 1915-1926.

31. Straus SE, Jaffe ES, Puck JM, Dale JK, Elkon KB, Rosen-Wolff A et al. The development of lymphomas in families with autoimmune lymphoproliferative syndrome with germline Fas mutations and defective lymphocyte apoptosis. Blood 2001; 98: 194-200.

32. Muschen M, Warskulat U, Beckmann MW. Defining CD95 as a tumor suppressor gene. J Mol Med (Berl) 2000; 78: 312-325.

33. Helmbach $H$, Rossmann E, Kern MA, Schadendorf D. Drug-resistance in human melanoma. Int J Cancer 2001; 93: 617-622.

34. Shin MS, Park WS, Kim SY, Kim HS, Kang SJ, Song KY et al. Alterations of Fas (Apo-1/CD95) gene in cutaneous malignant melanoma. Am J Pathol 1999; 154 1785-1791.

35. Ivanov VN, Bhoumik A, Krasilnikov M, Raz R, Owen-Schaub LB, Levy D et al. Cooperation between STAT3 and c-jun suppresses Fas transcription. Mol Cell 2001; 7: 517-528.
36. Peli J, Schroter M, Rudaz C, Hahne M, Meyer C, Reichmann E et al. Oncogenic Ras inhibits Fas ligand-mediated apoptosis by downregulating the expression of Fas. EMBO J 1999; 18: 1824-1831.

37. Santourlidis S, Warskulat U, Florl AR, Maas S, Pulte T, Fischer J et al. Hypermethylation of the tumor necrosis factor receptor superfamily 6 (APT1, Fas, CD95/Apo-1) gene promoter at rel/nuclear factor kappaB sites in prostatic carcinoma. Mol Carcinog 2001; 32: 36-43.

38. Sato T, Irie S, Kitada S, Reed JC. FAP-1: a protein tyrosine phosphatase that associates with Fas. Science 1995; 268: 411-415.

39. Krasilnikov M, Ivanov VN, Dong J, Ronai Z. ERK and PI3K negatively regulate STAT-transcriptional activities in human melanoma cells: implications towards sensitization to apoptosis. Oncogene 2003; 22: 4092-4101.

40. Medema JP, de Jong J, van Hall T, Melief CJ, Offringa R. Immune escape of tumors in vivo by expression of cellular FLICE-inhibitory protein. J Exp Med 1999; 190: 1033-1038.

41. Nastiuk KL, Krolewski JJ. FLIP-ping out: death receptor signaling in the prostate. Cancer Biol Ther 2008; 7: 1171-1179

42. Fulda S, Kufer MU, Meyer E, van Valen F, Dockhorn-Dworniczak B, Debatin KM. Sensitization for death receptor- or drug-induced apoptosis by re-expression of caspase-8 through demethylation or gene transfer. Oncogene 2001; 20: 5865-5877.

43. Dikic I, Wakatsuki S, Walters KJ. Ubiquitin-binding domains - from structures to functions. Nat Rev Mol Cell Biol 2009; 10: 659-671.

44. Rahighi S, Ikeda F, Kawasaki M, Akutsu M, Suzuki N, Kato R et al. Specific recognition of linear ubiquitin chains by NEMO is important for NF-kappaB activation. Cell 2009; 136: 1098-1109.

45. Raasi S, Varadan R, Fushman D, Pickart CM. Diverse polyubiquitin interaction properties of ubiquitin-associated domains. Nat Struct Mol Biol 2005; 12: 708-714.

46. Hofmann K, Bucher P. The UBA domain: a sequence motif present in multiple enzyme classes of the ubiquitination pathway. Trends Biochem Sci 1996; 21: 172-173.

47. Kirkin V, Lamark T, Sou YS, Bjorkoy G, Nunn JL, Bruun JA et al. A role for NBR1 in autophagosomal degradation of ubiquitinated substrates. Mol Cell 2009; 33: 505-516.

48. Song B, Xie B, Wang C, Li M. Caspase-3 is a target gene of c-Jun: ATF2 heterodimers during apoptosis induced by activity deprivation in cerebellar granule neurons. Neurosci Lett 2011; 505: 76-81.

49. Kaufmann T, Strasser A, Jost PJ. Fas death receptor signalling: roles of Bid and XIAP. Cell Death Differ 2011; 19: 42-50.

50. Ricci MS, Jin Z, Dews M, Yu D, Thomas-Tikhonenko A, Dicker DT et al. Direct repression of FLIP expression by c-myc is a major determinant of TRAIL sensitivity. Mol Cell Biol 2004; 24: 8541-8555.

51. Calvisi DF, Wang C, Ho C, Ladu S, Lee SA, Mattu S et al. Increased lipogenesis, induced by AKT-mTORC1-RPS6 signaling, promotes development of human hepatocellular carcinoma. Gastroenterology 2011; 140: 1071-1083.

52. Kharbanda S, Pandey P, Yamauchi T, Kumar S, Kaneki M, Kumar V et al. Activation of MEK kinase 1 by the $\mathrm{C}-\mathrm{Abl}$ protein tyrosine kinase in response to DNA damage. Mol Cell Biol 2000; 20: 4979-4989.

53. Gupta S, Barrett T, Whitmarsh AJ, Cavanagh J, Sluss HK, Derijard B et al. Selective interaction of JNK protein kinase isoforms with transcription factors. EMBO J 1996; 15: 2760-2770.

54. Stennicke HR, Salvesen GS. Biochemical characteristics of caspases-3, $-6,-7$, and -8 . J Biol Chem 1997; 272: 25719-25723.

55. Stennicke HR, Deveraux QL, Humke EW, Reed JC, Dixit VM, Salvesen GS. Caspase-9 can be activated without proteolytic processing. J Biol Chem 1999; 274: 8359-8362.

56. Zhu J, Nozell S, Wang J, Jiang J, Zhou W, Chen X. p73 cooperates with DNA damage agents to induce apoptosis in MCF7 cells in a p53-dependent manner. Oncogene 2001; 20: 4050-4057.

57. Campeau E, Ruhl VE, Rodier F, Smith CL, Rahmberg BL, Fuss JO et al. A versatile viral system for expression and depletion of proteins in mammalian cells. PLoS One 2009; 4: $e 6529$.

(c) (i) (2) Cell Death and Disease is an open-access journal (Ey wa sa published by Nature Publishing Group. This work is licensed under a Creative Commons Attribution-NonCommercialShareAlike 3.0 Unported License. The images or other third party material in this article are included in the article's Creative Commons license, unless indicated otherwise in the credit line; if the material is not included under the Creative Commons license, users will need to obtain permission from the license holder to reproduce the material. To view a copy of this license, visit http://creativecommons.org/licenses/ by-nc-sa/3.0/ 